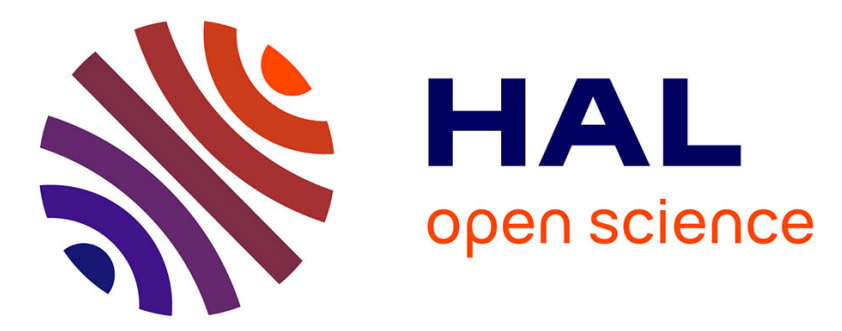

\title{
Characteristics of thoracic aortic aneurysm rupture in vitro
}

Yuanming Luo, Ambroise Duprey, Stéphane Avril, Jia Lu

\section{To cite this version:}

Yuanming Luo, Ambroise Duprey, Stéphane Avril, Jia Lu. Characteristics of thoracic aortic aneurysm rupture in vitro. Acta Biomaterialia, 2016, 42, pp.286 - 295. 10.1016/j.actbio.2016.06.036 . hal01380229

\section{HAL Id: hal-01380229 \\ https://hal.science/hal-01380229}

Submitted on 12 Oct 2016

HAL is a multi-disciplinary open access archive for the deposit and dissemination of scientific research documents, whether they are published or not. The documents may come from teaching and research institutions in France or abroad, or from public or private research centers.
L'archive ouverte pluridisciplinaire HAL, est destinée au dépôt et à la diffusion de documents scientifiques de niveau recherche, publiés ou non, émanant des établissements d'enseignement et de recherche français ou étrangers, des laboratoires publics ou privés. 


\title{
Characteristics of Thoracic Aortic Aneurysm Rupture in vitro
}

\author{
Yuanming Luo ${ }^{1}$, Ambroise Duprey ${ }^{2,3,4,5}$, Stéphane Avriil ${ }^{2,3,4}$, and Jia Lu*1 \\ ${ }^{1}$ Department of Mechanical \& Industrial Engineering, The University of Iowa, Iowa City, IA 52242, \\ USA \\ ${ }^{2}$ Ecole Nationale Supérieure des Mines de Saint-Etienne, CIS-EMSE, SAINBIOSE, F-42023 St. \\ Étienne, France \\ ${ }^{3}$ INSERM, U1059, F-42000 Saint Etienne, France \\ ${ }^{4}$ Université de Lyon, SAINBIOSE, F-42000 Saint Etienne, France \\ ${ }^{5}$ Cardiovascular Surgery Service, CHU de Saint-Etienne, F-42055 St. Étienne CEDEX 2, France
}

\begin{abstract}
Ascending thoracic aortic aneurysms (ATAAs) are focal dilatations in the aorta that are prone to rupture or dissection. To accurately evaluate the rupture risk, one must know the local mechanical conditions at the rupture site and understand how rupture is triggered in a layered fibrous media. A challenge facing experimental studies of ATAA rupture is that the ATAA tissue is highly heterogeneous; experimental protocols that operate under the premise of tissue homogeneity will have difficulty delineating the location conditions. In this work, we employed a previously established pointwise identification method to characterize wall stress, strain, and property distributions to a sub-millimeter resolution. Based on the acquired field data, we obtained the local mechanical properties at the rupture site in nine ATAA tissue samples. The rupture stress, ultimate strain, energy density, and the toughness of the tested samples were also reported. Our results show that the direction of the rupture is aligned with the direction of maximum stiffness, indicating that higher stiffness is not always related to higher strength. It was also found that the rupture generally occurs at a location of highest stored energy. As a higher stiffness and higher strain energy indicate a larger recruitment of collagen fibers in the tissue at the location and along the direction of rupture, the recruitment of collagen fibers in the deformation of the tissue is probably essential in ATAA rupture.
\end{abstract}

Keywords: Thoracic Aortic Aneurysms; Rupture; Rupture pattern; Rupture stress; Toughness

${ }^{*}$ Corresponding author. E-mail: jia-lu@ uiowa.edu 


\section{Introduction}

Ascending thoracic aortic aneurysms are focal dilatations of the aortic wall $[6,11]$. They may grow silently or may suddenly rupture or dissect, causing life-threatening internal bleeding. Studies have shown that the likelihood of rupture or dissection is greater than $30 \%$ when an aneurysm's diameter exceeds $6.0 \mathrm{~cm}$ [11]. The mortality rate of ruptured thoracic aortic aneurysms is nearly $100 \%[6,17]$. Currently surgical intervention is indicated when the diameter of ATAAs is greater than $5.5 \mathrm{~cm}$ [4]. While size is a common criterion, there is a need for identifying better indicators in monitoring and evaluating ATAAs. From the standpoint of biomechanics, rupture propensity hinges on local mechanical conditions and micromechanical events leading to fracture initiation. However, our current knowledge on these subjects remains poor.

Several studies have investigated the mechanical properties, including rupture strength, of ATAA tissues. Vorp et al [34], Iliopoulos et al [16], Choudhury et al [5] and Khanafer et al [19] measured the tensile properties using uni-axial tensile tests. They examined the regional and directional differences using specimens harvested from different locations and orientations, and reported moderate to significant differences in both stiffness and strength. Okamoto et al [24], Matsumoto et al [23], Azadani et al [1] and Pham et al [25] investigated the bi-axial properties using bi-axial tension tests. They also reported regional and directional properties, and compared the properties of ATAAs caused by various diseases [23] or of different age groups [24]. Martin et al [22] developed a predictive model to assess the in vivo rupture risk of ATAAs based on the measured strength and estimated wall stress. Sugita et al [31] used an inflation test to measure the bi-biaxial properties, and reported a strong correlation between strength and a characteristic stiffness modulus [30]. On the related subject of abdominal aortic aneurysm (AAA) strength, Vorp's group $[28,33]$ measured the tensile strength of AAA tissues. Vande Geest et al [32] developed a statistical model for estimating the distribution of AAA wall strength considering aneurysm size, local diameter, and local intraluminal thrombus thickness as well patient's age, gender, family history of AAA, smoking status. Regional variability of AAA tensile strength was reported in AAAs [27]. It was found that the strength of specimen strips at or close to rupture sites was mostly low.

One of the challenges in the experimental study of ATAA properties is that the ATAA tissues are heterogeneous. ATAAs undergo continuous remodeling, and the properties are modulated by the local cellular activities underneath the pathological development $[2,18]$. Recent studies $[7,8]$ by the authors indicated that there is a significant level of heterogeneity in ATAA samples of centimeter dimension, a typical size used in the aforementioned mechanical tests. When the material is not reasonably homogeneous, these tests no longer generate a uniform stress field in the center region of specimen. Thus, the measured stress and strain, which are ho- 
mogenized values, may not truly reflect the local values at the rupture site. For this and other reasons, it is not surprising that conflicting results were reported on the regional and directional stiffness and strength. In this regard, a fundamental limitation of the previous studies is the underlying assumption of tissue homogeneity. The assumption may be appropriate for estimating the global elastic properties, but not the local rupture conditions. Motivated by the need of delineating the local conditions, our laboratories have developed a method that can identify the tissue properties to a sub-millimeter resolution [7,8]. This method integrated Digital Image Correlation (DIC), inflation test and an inverse stress analysis methodology [21], enabling the identification of full-field stress, strain and mechanical properties without being limited by the complexity of the tissue heterogeneity. In a parallel study along the same line, a different analysis approach was employed [29]. We have characterized the heterogeneous anisotropic properties of ten ATAA samples [8]. The peak tension was also determined in all these tests. The availability of the field data enabled the investigation of the local conditions at the rupture sites and the exploration of possible link between elastic properties and rupture. In this paper, we report these findings.

\section{Methods}

\subsection{Experiment}

Nine ATAA sections were collected from seven patients undergoing elective surgery to replace their ATAA with a graft in accordance with a protocol approved by the Institutional Review Board of the University Hospital Center of St. Étienne and then tested according to our previously developed protocol for identifying the pointwise distribution of the mechanical properties of soft tissues using bulge inflation tests [7]. Patients' information is briefly summarized in Table 1 .

Specimens of approximately $4 \times 4 \mathrm{~cm}^{2}$ cut from the excised sections were clamped to an inflation device and inflated using water at a constant rate until rupture. Images of the outer surface were recorded every $3 \mathrm{kPa}$. Three dimensional displacement of the tissue surface was extracted using a commercial DIC software, ARAMIS (GOM, v. 6.2.0). A deforming NURBS surface that corresponds through all pressure states were derived from the DIC point clouds for the center region of each specimen. The size of mesh region is approximately $2.5 \times 2.5 \mathrm{~cm}^{2}$. The surface deformation tensor, $\mathbf{C}$, and the Cauchy-Green strain, $\mathbf{E}$, were computed from the deforming NURBS meshes. The surface tension (the resultant stress over the wall thickness) at every Gauss point was computed using the inverse method in [21], which solved the equilibrium problem directly on a deformed 


\begin{tabular}{r|cccc}
\hline Patient & Gender(M/F) & Age & Diameter(mm) & Thickness(mm) \\
\hline 1 & M & 55 & 55 & 2.35 \\
2 & F & 65 & 49 & 1.82 \\
3 & F & 80 & 52 & 1.68 \\
4 & M & 79 & 52 & 1.76 \\
5 (a) \& (b) & M & 76 & 58 & 1.82 \\
6 & M & 72 & 51 & 1.90 \\
$7(\mathrm{a})$ & F & 76 & 65 & 2.38 \\
$7(b)$ & F & 76 & 65 & 2.51 \\
\hline
\end{tabular}

Table 1: Sample Information

configuration. Having obtained the stress and strain distributions at every pressure steps, the stress-strain curves at every Gauss point were collected. The stress-strain data were then fitted to a hyperelastic constitutive equation to identify the material parameters. The theoretical underpinnings and additional details of this pointwise identification approach can be found in $[13,20,35,36]$.

A material model was adopted from the work of Gasser, Ogden, and Holzapfel $(\mathrm{GOH})[12,14]$ to describe the planar response of the ATAA tissue:

$$
w=\frac{\mu_{1}}{2}\left(\mathrm{I}_{1}-\ln \mathrm{I}_{2}-2\right)+\frac{\mu_{2}}{4 \gamma}\left(\mathrm{e}^{\gamma\left(\mathrm{I}_{\kappa}-1\right)^{2}}-1\right)
$$

The tissue is modeled as a single layer composite material consisting of a matrix and angularly distributed fibers. The first term represents the response of elastin network and ground substances, and $\mathrm{I}_{1}=\operatorname{tr} \mathbf{C}$ and $\mathrm{I}_{2}=\operatorname{det} \mathbf{C}$ are the principal invariants of the deformation tensor. The second term, which dominates the energy, represents the contribution of collagen fibers, with $\mathrm{I}_{k}$ being an anisotropic strain invariant

$$
\mathrm{I}_{\kappa}=\kappa \mathrm{I}_{1}+(1-2 \kappa) \mathbf{M} \cdot \mathbf{C M}
$$

In obtaining this invariant the fibers are assumed symmetrically distributed with respect to two mutually perpendicular directions. The parameter $\kappa$ represents the dispersion of the angular distribution. The value of $\kappa$ varies from 0 to 0.5 . When $\kappa=0$ all of the collagen fibers are perfectly aligned in the direction of $\mathbf{M}$ and when the fibers are uniformly distributed (isotropy), $\kappa=0.5$. For $0<\kappa<0.5$, the model is planar orthotropic with $\mathbf{M}$ being the major symmetry axis. The vector $\mathbf{M}$ will be called the fiber direction in the sequel. It should be 
noted that, unless $\kappa=0$, this vector is not a bona fide fiber direction but the direction of the predominate distribution and consequently the highest stiffness. The vector $\mathbf{M}$ is parameterized by the angle it makes relative to the horizontal axis, and the angle was identified along with other constitutive parameters. When $\kappa=0.5$, the angle becomes meaningless. This model was proved sufficient to recover the macroscopic behavior of the tissue $[7,8]$.

\subsection{Rupture site and rupture tension}

The rupture tension is defined as the ultimate tension at the rupture site. Since it is unclear whether a component of the tension or the norm (i.e. the total) is a better index, both the norm and the component in the orifice normal direction are reported. The rupture site was identified from the photo image of the second last loading step (the one immediate preceding rupture), or the post rupture image if the one before does not show distinct cracks. The identification scheme is illustrated in Figure 1. The bounding coordinates of the orifice were measured in the DIC camera coordinate system. The average values of the horizontal and vertical directions were assumed to be the location of rupture initiation, that is the rupture site. Most specimens bore narrow elliptical cracks. There were two cases having symmetric $\wedge$-shaped or curved orifices. For these cases, the center of symmetry was taken to be the rupture site. The scheme could induce uncertainties in the identified location. To alleviate the influence, the average tension in a small window containing the rupture site was used to determine the rupture tension. The window consists of a patch of $3 \times 3$ elements with the crack element in the middle, surrounded by eight elements (Figure1(b)). This $3 \times 3$ configuration is the second smallest topologically symmetric area in the hierarchy of mesh geometry. The size of the window is approximately $9 \mathrm{~mm}^{2}$. The same average was applied to all other rupture site variables.

\subsection{Orifice orientation}

In seven out of nine cases, the orifices were narrow straight slivers, enabling an easy definition of orientation. Figure 1(c) illustrates how the orientation was determined. The direction of crack propagation was manually identified, from which the normal direction was determined following the right-hand rule. For the $\wedge$-shape orifices (Figures 3 and $9(\mathrm{k})$ ), each branch was treated as a single straight crack. The orientation of the orifice was characterized by the angle the normal makes to the horizontal direction. 


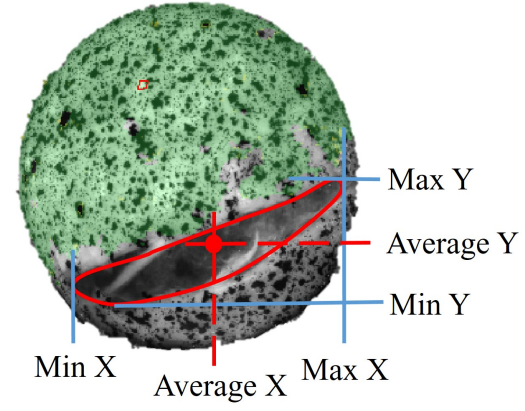

(a) A post rupture image showing orifice

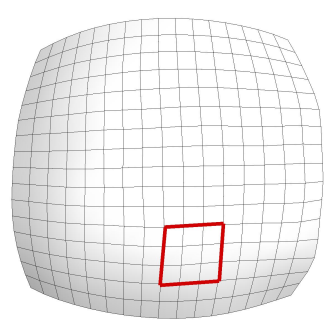

(b) Local window

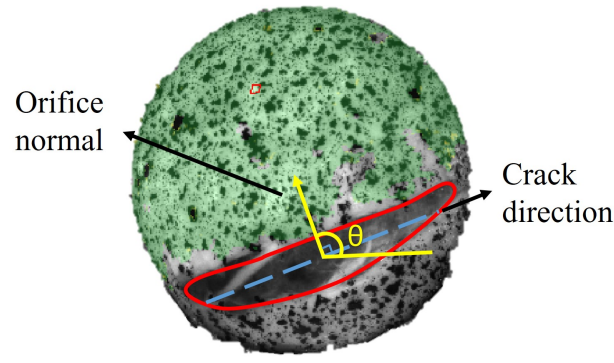

(c) Orifice orientation

Figure 1: Determination of rupture site and orifice orientation

\subsection{Toughness}

Toughness, a measure of material's resistance to fracture, is the amount of energy needed to fracture a material. It can be computed by accumulating the work done by the stress prior to fracture, viz. $T H=\int_{\mathbf{0}}^{\mathbf{E}_{f}} T_{I J} d E_{I J}$. Here $T_{I J}$ and $E_{I J}$ are the components of the second Piola-Kirchhoff tension and Cauchy-Green strain, respectively, $\mathbf{E}_{f}$ is the ultimate strain, and $T H$ is the toughness. As the tension and strain values were made available at each Gauss point throughout the loading process, the stress work can be computed locally. A middle points rule was used for this computation:

$$
W \approx \sum_{k=1}^{n s-1} \frac{\left(T_{I J}^{(k)}+T_{I J}^{(k+1)}\right)\left(E_{I J}^{(k+1)}-E_{I J}^{(k)}\right)}{2}
$$

where $T_{I J}$ is a component of the second Piola-Kirchhoff tension, $E_{I J}$ is a component of the surface strain, and $n s$ is the number of loading steps. The stress work at the rupture site was used to determine the toughness. Again the values were computed by averaging the stress work in the local window.

\section{Results}

\subsection{Orifice orientation vs fiber direction}

In each specimen the fiber angle at the rupture site was identified, and then compared to the orifice orientation. Figure 2 presents the result. A sharp linear relation $(y=x)$ between the fiber angle and the orifice normal angle is observed, indicating that the orifice is perpendicular to the local fiber direction.

In addition, the direction of rupture propagation appears to correlate with the fiber direction as well. In a significant number of specimens the orifices appear to be preferentially transverse. This directional preference 


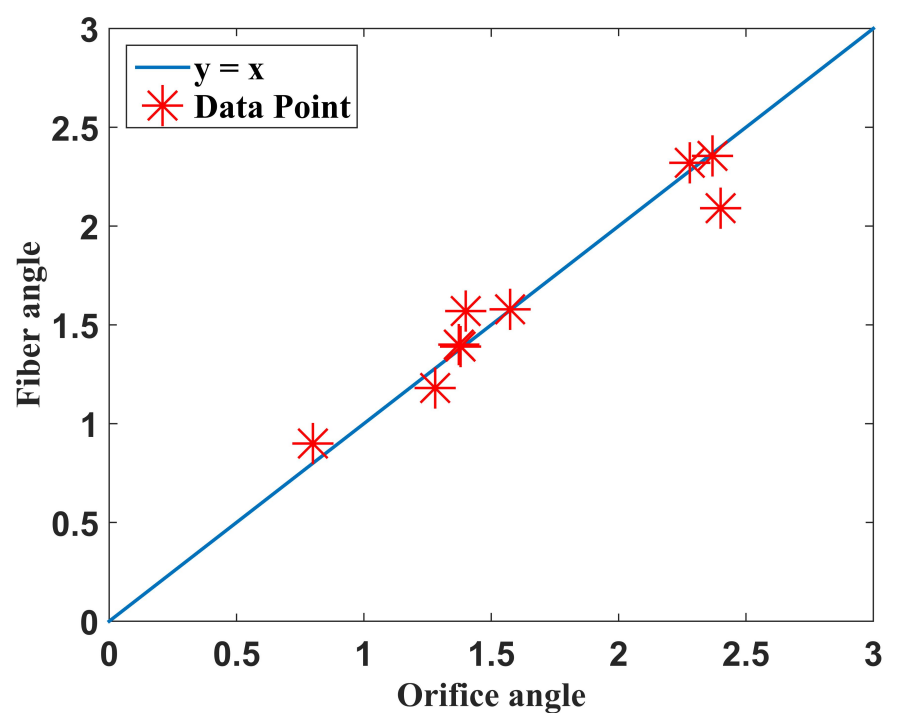

Figure 2: Predicted fiber direction vs orifice normal at rupture sites

manifests itself most strikingly in curved cracks, as shown in Figure 3. The red (gray in black-white print) crescent in the post-rupture image outlines the orifice. The crescent is superimposed on the fiber distribution map, at the same location with respect to the image coordinate system. It is shown that the fiber vectors in the orifice region are mostly transverse to the orifice. All but one sample were found to follow this normality rule, and seven additional cases are shown in Figure 9 in the Appendix. The outlier will be discussed later. It should be emphasized that the fiber angle (or direction) is a model parameter, not the physical angle of fiber bundles. The angle was predicted from the acquired tension-strain data, not directly measured.

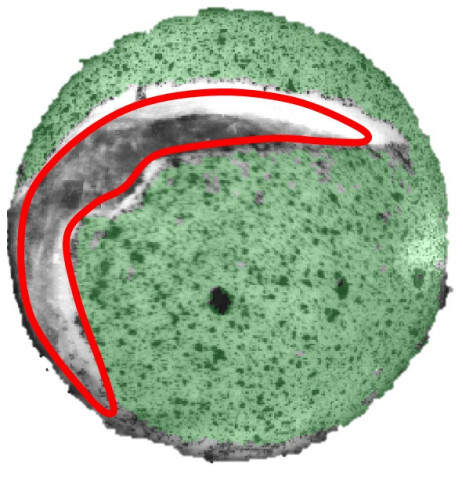

(a) Post-rupture image

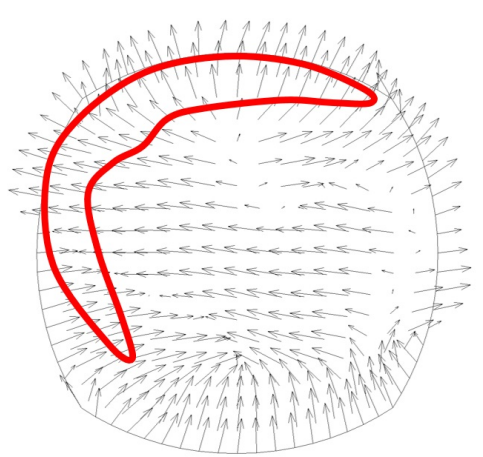

(b) Predicted fiber direction

Figure 3: Predicted fiber orientation and the direction of fracture propagation

Recall that in the GOH model the fiber vector $\mathbf{M}$ represents the direction of the highest stiffness. To verify whether the fiber direction was correctly described, we examined the directional stiffness of the tissue. The 
tension and strain at the rupture site were rotated to a local system in which the $\mathbf{e}_{1}$ basis coincides with the fiber direction and $\mathbf{e}_{2}$ the transverse direction. The rotated tension-strain curves of all cases were generated and examined. An example of such curves is shown in Figure 4. Note that the tension state is bi-axial; any component (say $T_{11}$ ) is a function of three strain components. The response functions are surfaces in a high dimensional space. The curves shown here are the projections of the response function into two-dimensional spaces. In all cases the $T_{11}$ curve lies above $T_{22}$, indicating that the tension in the fiber direction is higher than that in the transverse direction in all load steps. Given that the strains are approximately equal-biaxial, the fact that $T_{11}$ is above $T_{22}$ indicates that the tissue is indeed stiffer in the identified fiber direction.

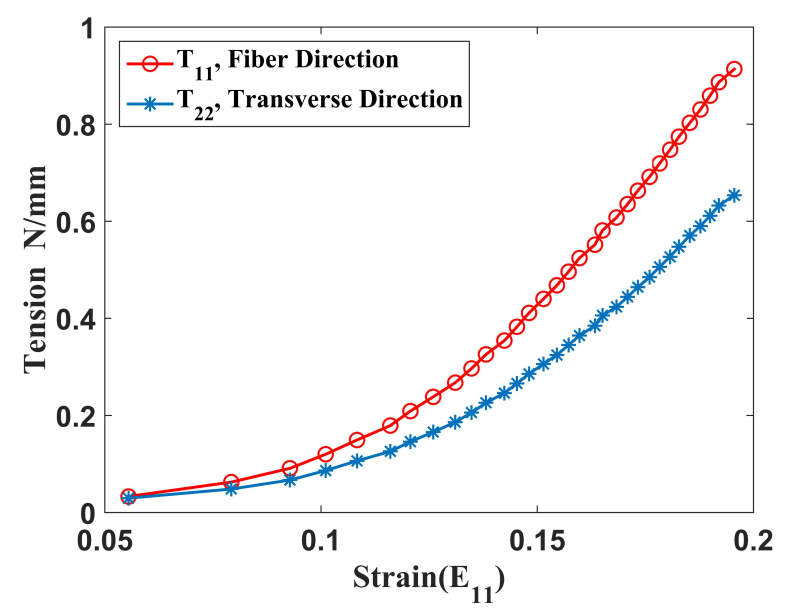

(a) Patient 2: $T_{11}$ and $T_{22}$ vs $E_{11}$

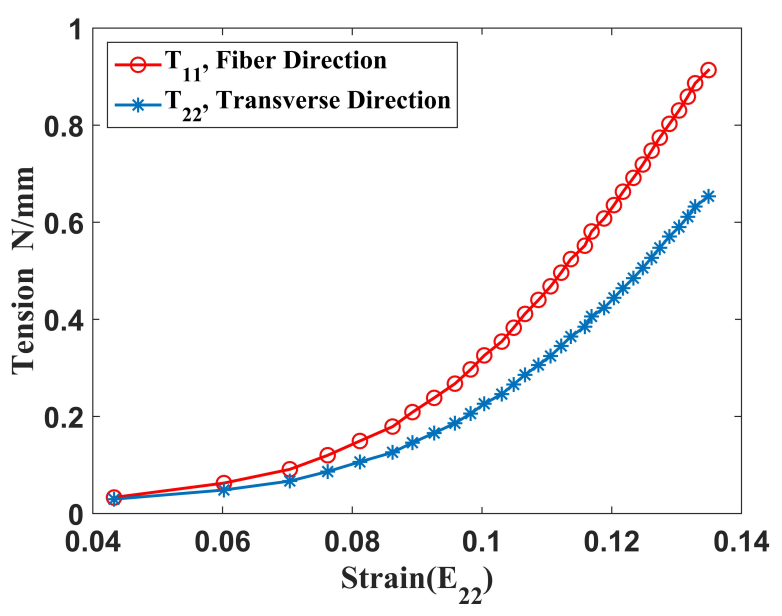

(b) Patient 2: $T_{11}$ and $T_{22}$ vs $E_{22}$

Figure 4: Tension-strain curves in the predicted fiber and the transverse directions

Could it be that $T_{11}$ happens to be the principal tension so that the tissues were merely cleaving in the direction of maximum normal tension? We examined the principal directions and found that they do not coincide with the fiber direction in several cases. An example is shown in Figure 5. The principal directions were computed from the state prior to rupture. It is clear that the fiber directions are different from the principal directions. In particular, the principal direction is parallel to the orifice in the crack zone, indicating that the tissue did not fracture in the direction of maximum normal tension.

\subsection{Strength and toughness}

The norm of the rupture tension, $T_{f}$, the component in the fiber direction, $T_{n}$, the ultimate strain (norm), $E_{f}$, the component in the fiber direction, $E_{n}$, and the toughness TH are listed in Table 2. Note that the fiber direction can also be interpreted as the direction of orifice normal at rupture site. No significant relation is found between 


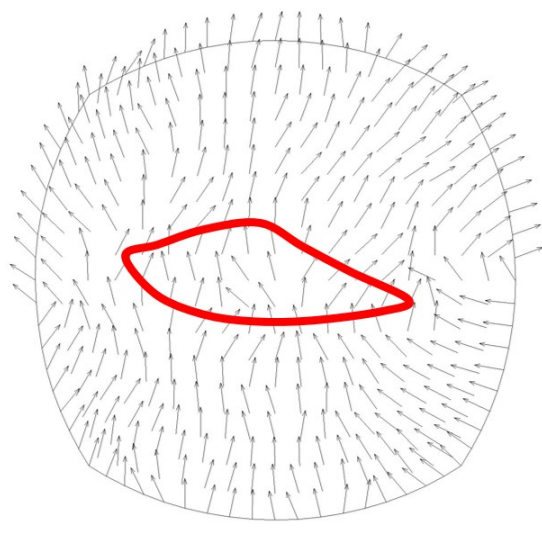

(a) Predicted fiber direction

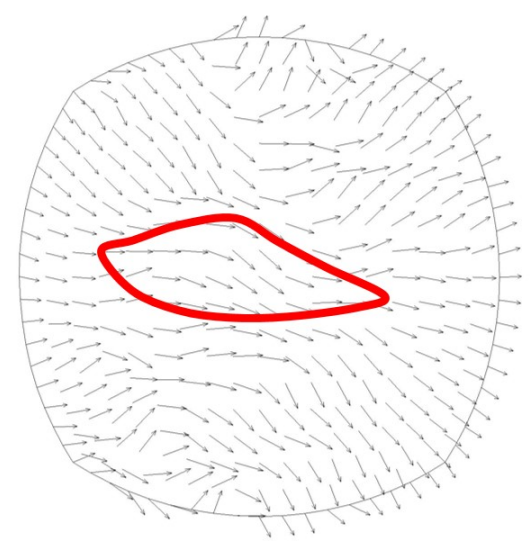

(b) Principal direction

Figure 5: Comparison of the predicted fiber direction and principal tension direction. In the crack zone the principal tension aligns with the orifice

$T_{f}$ and TH $(\mathrm{p}=0.008)$, between $T_{n}$ and TH $(\mathrm{p}=0.01)$, between $E_{f}$ and $\mathrm{TH}(\mathrm{p}=1.75 \mathrm{E}-06)$ and between $E_{n}$ and $\mathrm{TH}(\mathrm{p}=3.45 \mathrm{E}-06)$.

\begin{tabular}{r|ccccc}
\hline Patient & $T_{f}(\mathrm{~N} / \mathrm{mm})$ & $T_{n}(\mathrm{~N} / \mathrm{mm})$ & $E_{f}$ & $E_{n}$ & $\mathrm{TH}(\mathrm{N} / \mathrm{mm})$ \\
\hline 1 & 1.71 & 1.32 & 0.90 & 0.60 & 0.34 \\
2 & 1.12 & 0.91 & 0.26 & 0.19 & 0.07 \\
3 & 0.64 & 0.51 & 0.21 & 0.16 & 0.04 \\
4 & 1.44 & 1.08 & 0.30 & 0.19 & 0.10 \\
$5(\mathrm{a})$ & 0.75 & 0.56 & 0.38 & 0.25 & 0.07 \\
$5(\mathrm{~b})$ & 0.62 & 0.52 & 0.16 & 0.09 & 0.02 \\
6 & 0.48 & 0.37 & 0.21 & 0.13 & 0.04 \\
$7(\mathrm{a})$ & 1.13 & 1.00 & 0.26 & 0.16 & 0.07 \\
$7(\mathrm{~b})$ & 0.37 & 0.31 & 0.14 & 0.11 & 0.02 \\
\hline
\end{tabular}

Table 2: Strength, ultimate strain, and toughness

To explore the correlation between rupture site and the peak tension or peak energy, we superimpose the identified rupture window on the tension and energy contours. Figure 6 presents two cases. The remaining seven cases are included in Figure 10 in the Appendix. The boxes are the local window defining the rupture site. It can be seen that for patient 1 , both the peak tension and the peak energy coincide with the rupture location. For 
patient 2, however, the peak energy coincides with the rupture location while the peak tension does not. When the local rupture window encloses or overlaps the peak tension or peak stress red spots, we say that a match is found. The result of matching for all nine cases is reported in Table 3. It can be seen that the peak energy matches in six cases, whereas the peak tension matches in four cases.

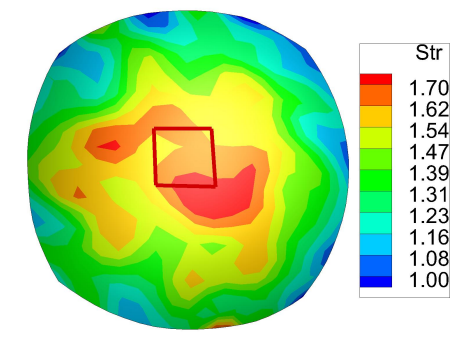

(a) Patient 1: Tension

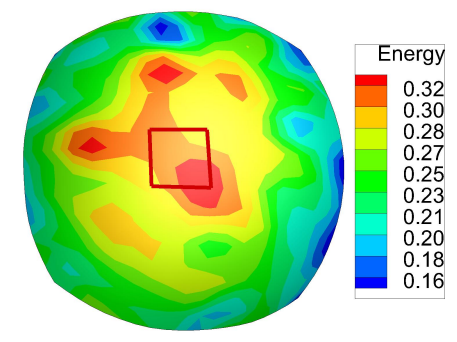

(b) Patient 1: Energy

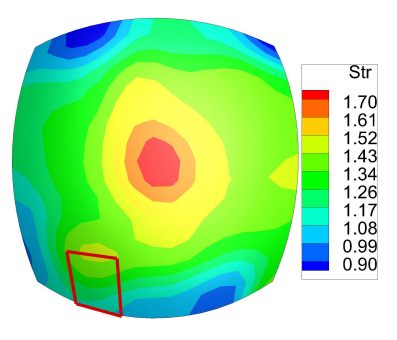

(c) Patient 2: Tension

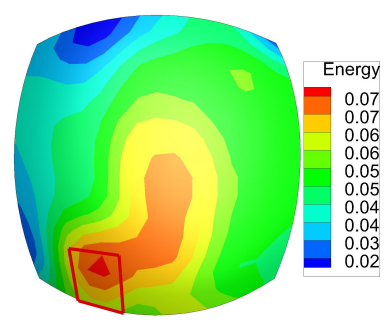

(d) Patient 2: Energy

Figure 6: Rupture site vs the locations of peak tension and peak energy

\begin{tabular}{c|ccccccccc}
\hline Patient & 1 & 2 & 3 & 4 & $5(\mathrm{a})$ & $5(\mathrm{~b})$ & 6 & $7(\mathrm{a})$ & $7(\mathrm{~b})$ \\
\hline Energy $(\mathrm{Y} / \mathrm{N})$ & $\mathrm{Y}$ & $\mathrm{Y}$ & $\mathrm{N}$ & $\mathrm{Y}$ & $\mathrm{Y}$ & $\mathrm{N}$ & $\mathrm{Y}$ & $\mathrm{Y}$ & $\mathrm{N}$ \\
Tension $(\mathrm{Y} / \mathrm{N})$ & $\mathrm{Y}$ & $\mathrm{N}$ & $\mathrm{N}$ & $\mathrm{Y}$ & $\mathrm{Y}$ & $\mathrm{N}$ & $\mathrm{N}$ & $\mathrm{Y}$ & $\mathrm{N}$ \\
\hline
\end{tabular}

Table 3: Rupture location vs the position of peak tension or peak energy: "Y" = match, and "N" = no match

\section{Discussion}

Most biomechanical studies on ATAA properties treated tested specimens as homogeneous. Mechanical properties were obtained from samples of centimeter size. Recent studies $[7,8]$ by the authors showed that ATAA tissues are highly heterogeneous even at this size scale. At the presence of strong heterogeneities, homogenized stress and strain obtained from the uni-axial or bi-axial tests at centimeter scale may not be conclusive with regard to the local condition at rupture sites. To address this challenge, we investigated ATAA rupture characteristics at a millimeter scale. We identified the stress, strain, and properties distributions to within a submillimeter resolution. Based on field data of this resolution, we obtained rupture site values by averaging the field data in a local window of approximately $3 \times 3 \mathrm{~mm}^{2}$. At this level of locality, the ATAA's rupture appears to exhibit some distinct characteristics and consistent patterns.

The most striking pattern is that the cracks are preferentially transverse to the fiber direction - the direction of 
maximum stiffness. In all but one case the orifice normal is parallel to the local fiber direction. The cracks appear to propagate in the same pattern, resulting in curved orifices in some cases. The only exception to this directional pattern is patient 4 , for which the post-rupture image, the fiber map and the contour of the dispersion parameter $\kappa$ are shown in Figure 7. The orifice is roughly a prolate oval, seemingly parallel to the "fiber direction". A close look at the $\kappa$ contour suggests that this sample is nearly isotropic in the center region where the crack initiated. It can be seen from Figure $7(\mathrm{c})$ that the $\kappa$ value around the initial rupture site (the windowed region) is in the neighborhood of 0.47 . Recall that a $\kappa$ value of 0.5 indicates isotropy. The tension-strain curves in the fiber and transverse directions are very close (Figures 7(d) and (e)), confirming a near isotropy. Comparing with other cases this sample has the highest average $\kappa$ value. When the material is nearly isotropic, the "fiber direction" becomes less meaningful, if not completely meaningless. Thus, this case is inconclusive. The seemingly conflicting orientation does not contradict the other cases. In contrast, it highlights the underlying role of fiber distribution.

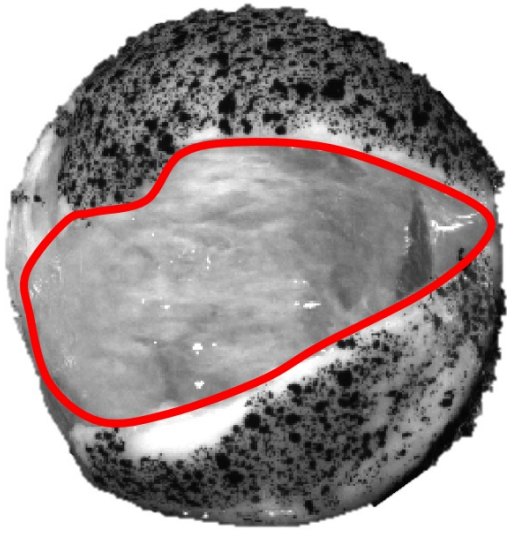

(a) Post-rupture image

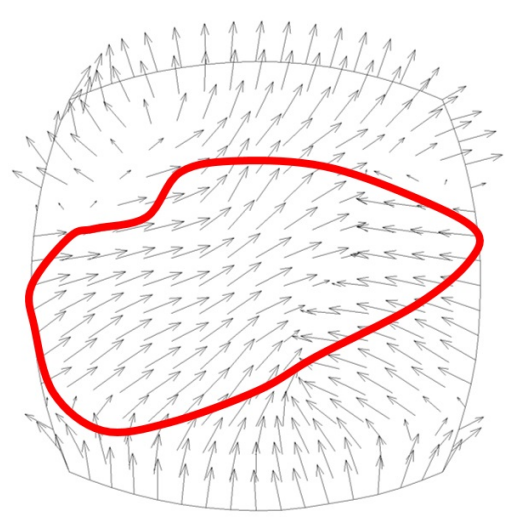

(b) Predicted fiber direction

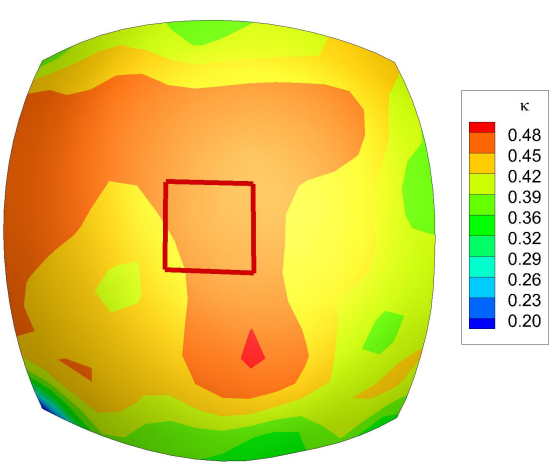

(c) Dispersion parameter $\kappa$

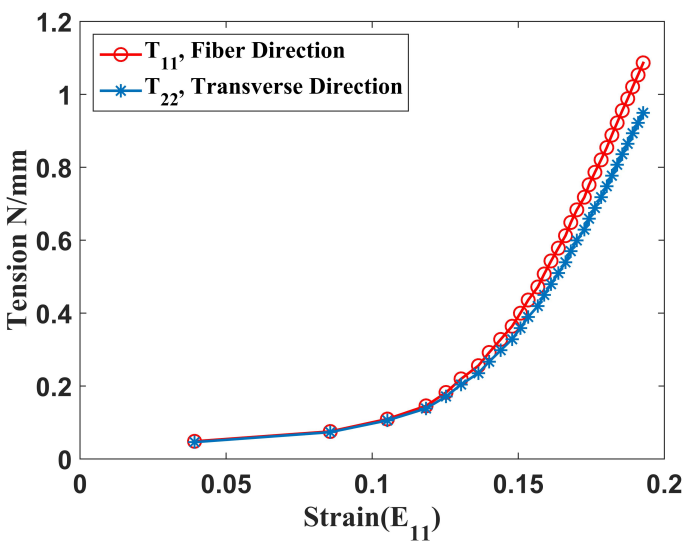

(d) $T_{11}$ and $T_{22}$ vs $E_{11}$

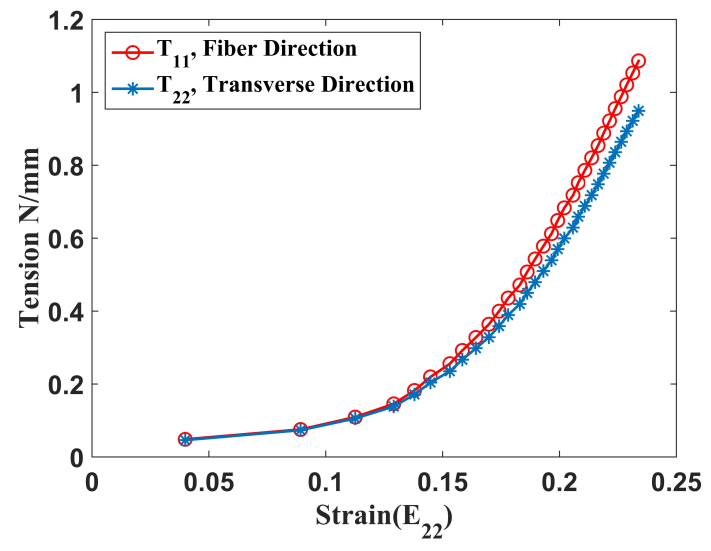

(e) $T_{11}$ and $T_{22}$ vs $E_{22}$

Figure 7: Orifice, fiber map, $\kappa$ contour and Tension strain curves at the rupture site for patient 4 
The observed directional preference has some implications. First, it is suggestive, albeit indirectly, of the rule of collagen microstructure in regulating ATAA tissue strength. Since the direction of maximum stiffness is the direction of predominate collagen recruitment, the present finding suggests that collagen recruitment is an important determinant of strength. This finding is also in line with the observation by Pichamuthu et al. [26], who noted that differences in mechanical properties are not attributable to absolute collagen content but may be accounted for by microstructural changes in the collagen framework. It has been long known that collagen turnover is important for vessel wall repair and its degradation is believed to be associated with the rupture of aortic aneurysms $[2,15,18]$. Recently, some studies revealed structural anomalies in the collagen network of aneurysmal tissues. Figueiredo Borges et al [9] reported that collagen is reduced and disrupted in human aneurysms and dissections of ascending aorta. They found that while appearing as uniform lamellae in the control group, the collagen fibers are disrupted and irregular in ATAA tissues. Carmo et al. [3] found that the collagen content in abdominal aortic aneurysms is significantly lower than in controls, but there is a significant increase of collagen cross-links. They suggested that in aneurysmal aortic walls old collagen accumulates cross-links while new collagen biosynthesis is somehow defective. Clearly, there is a need to investigate the correlation between the microstructure characteristics of collage network and rupture response.

Secondly, an anisotropic rupture criterion is needed for correctly predicting the crack initiation and propagation. A criterion based on the maximum principal stresses is unlikely to be adequate because it does not account for the possible directional strength and biaxial coupling effects. Further work is needed to establish anisotropic rupture criterion appropriate to ATAA tissues.

The correlation between the energy concentration and the rupture location is also worth-noting. The curves in Figure 8 show two distinct tension-strain behaviors at rupture sites. Figure 8(a) presents a case wherein the tension at the rupture site is consistently higher than a randomly chosen, non-rupture site, over a similar strain range. A possible explanation of this result could be that local fragmentation or loss of elastin induces an earlier recruitment of collagen locally. This earlier recruitment means stiffer properties, locally high stress and higher strain energy. Figure 8(b) demonstrates a different behavior whereby the tension at the rupture site is lower than a non-rupture site but the ultimate strain is much higher, resulting a higher energy. These cases suggest that, when assessing rupture propensity at tissue scale, both stress and strain should be concurrently considered. This conjecture is supported by a recent finding of Romo et al [29]. They showed that rupture of the ATAA does not systematically occur at the location of maximum stress, but at a weakened zone where strain localization happened. 


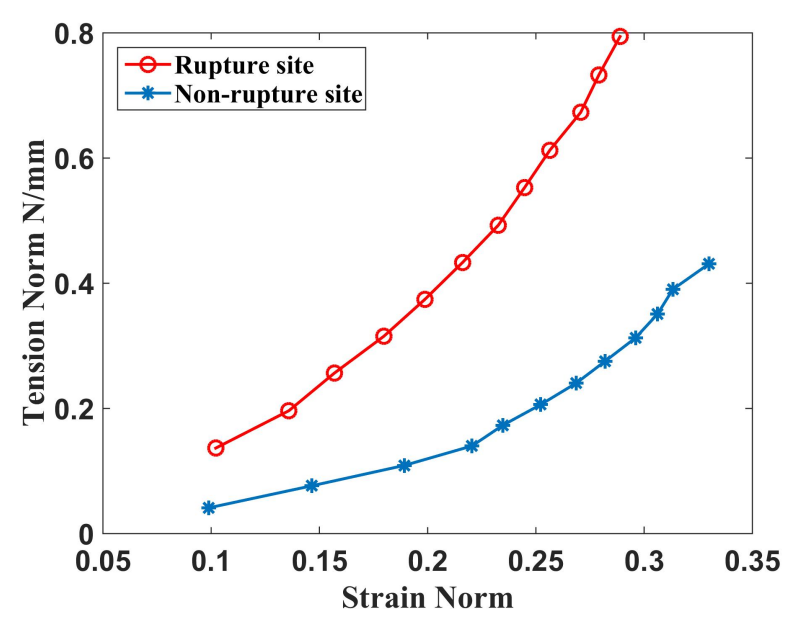

(a) Both energy and tension criteria worked as predictors of rupture

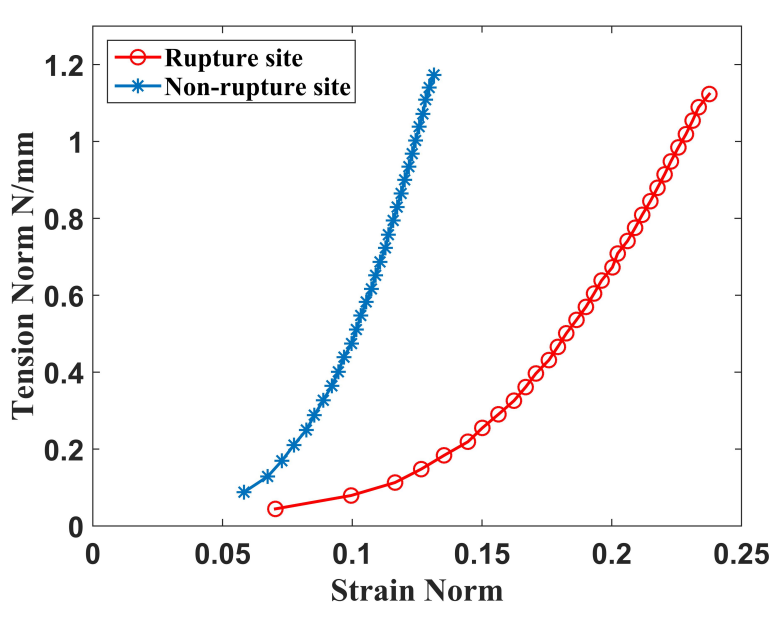

(b) Energy criteria worked as predictor of rupture

Figure 8: Two different stress-strain curves at rupture site and comparison to non-rupture sites

The study embodied several limitations. First, all findings were based on macroscopic descriptions. The "fiber direction" is a model representation of structural features, but not a direct physical quantity measured from instruments. Although a strong relation was revealed between the fiber direction and rupture angle, the actual fiber structure was not examined. Future work is needed to link microstructural features, if any, to the macroscopic behavior. There is a pressing need to image the collagen microstructures in order to understand the mechanisms underlying this directional preference. Secondly, the layered structure of the ATAA wall was not considered. The wall of the ATAA is composed of three distinct layers: the intima, media, and adventitia; each has different characteristics of collagen content and distribution. It has been reported that aortic dissection mostly initiates in the intima and media layers [10], indicating that the layered structure may play a role in determining the process of tissue failure. The interplay between the layers and rupture was not investigated. Moreover, there are no controls in the study, and thus it is unclear whether healthy ascending aorta follows the same or similar rupture pattern. The location of crack initiation was assumed at the geometric center of cracks identified from post-rupture images. This assumption could have been verified using a high-speed camera, which we plan to implement in future work. However, since the directional pattern is so pronounced as shown in Figures 3 and 9, slight or even moderate variations in the crack site location will not change the linear correlation shown in Figure 2. In parallel, the rupture strength and toughness were averaged from a local window of $3 \times 3$ elements. This would induce a certain level of uncertainties to the numerical values reported in Table 2. Nonetheless, the finding in Table 3 is unlikely to be affected in any significant manner if the window size is slightly different, as seen from the contours in Figures 6 and 10. It should also be noted that wall tension (the stress resultant 
over the thickness) was reported instead of the stress, because the wall thickness was not measured at enough points. Related to this, the stress work gives the energy density per unit surface area, not per unit volume as typically specified in mechanics. Lastly, the data presented are limited by the small population size. More work is needed to confirm the observations.

In summary, the present study revealed some distinct features of ATAA rupture in vitro under the inflation protocol. The rupture appears to initiate at the position when the surface energy density reaches a threshold value. The tissue fractures preferentially in the direction of the highest stiffness, generating orifices that are locally transverse to the fiber direction. In the future, we plan to conduct imaging studies on ATAA microstructure, combining our macroscopic analysis with microstructural interrogation. This multiscale approach may shed light on understanding the mechanisms of ATAA rupture, and hopefully may help to develop better means of risk assessment.

Acknowledgment. The authors would like to thank Drs. Aaron Romo and Frances M. Davis for their contributions in generating the inflation test data used in this study.

\section{Appendix}

\section{Fiber orientation versus orifice angle}

Figure 9 placed here

\section{Rupture site versus the locations of peak tension and peak energy}

Figure 10 placed here

\section{References}

[1] Ali N Azadani, Sam Chitsaz, Alex Mannion, Aart Mookhoek, Andrew Wisneski, Julius M Guccione, Michael D Hope, Liang Ge, and Elaine E Tseng. Biomechanical properties of human ascending thoracic aortic aneurysms. Annals of Thoracic Surgery, 96:50-58, 2013. 
[2] Panagiotis Berillis. The role of collagen in the aortas structure. The Open Circulation and Vascular Journal, pages 1-8, 2013.

[3] M. Carmo, L. Colombo, A. Bruno, F. R. M. Corsi, L. Roncoroni, M. S. Cuttin, F. Radice, E. Mussini, and P. G. Settembrini. Alteration of elastin, collagen and their cross-links in abdominal aortic aneurysms. Eur J Vasc Endovasc Surg, 23:543-549, 2002.

[4] Katherine H Chau and John A Elefteriades. Natural history of thoracic aortic aneurysms: size matters, plus moving beyond size. Prog. Cardiovasc. Dis., 56:74-80, 2013.

[5] Nusrat Choudhury, Olivier Bouchot, Leonie Rouleau, Dominique Tremblay, Raymond Cartier, Jagdish Butany, Rosaire Mongrain, and Richard L. Leask. Local mechanical and structural properties of healthy and diseased human ascending aorta tissue. Cardiovascular Pathology, 18:83-91, 2009.

[6] Ryan R Davies, Lee J Goldstein, Michael A Coady, Shawn L Tittle, John A Rizzo, Gary S Kopf, and John A Elefteriades. Yearly rupture or dissection rates for thoracic aortic aneurysms: simple prediction based on size. The Annals of thoracic surgery, 73(1):17-28, 2002.

[7] Frances M Davis, Yuanming Luo, Stéphane Avril, Ambroise Duprey, and Jia Lu. Pointwise characterization of the elastic properties of planar soft tissues: application to ascending thoracic aneurysms. Biomechanics and Modeling in Mechanobiology, 14:967-978, 2015.

[8] Frances M Davis, Yuanming Luo, Stéphane Avril, Ambroise Duprey, and Jia Lu. Local mechanical properties of human ascending thoracic aneurysms. Journal of the Mechanical Behavior of Biomedical Materials, 2016 (in press).

[9] Luciano de Figueiredo Borges, Rodrigo Gibin Jaldin, Ricardo Ribeiro Dias, Noedir Antonio Groppo Stolf, Jean-Baptiste Michel, and Paulo Sampaio Gutierrez. Collagen is reduced and disrupted in human aneurysms and dissections of ascending aorta. Human Pathology, 39:437-443, 2008.

[10] Roman W DeSanctis, Robert M Doroghazi, W Gerald Austen, and Mortimer J Buckley. Aortic dissection. New England Journal of Medicine, 317(17):1060-1067, 1987.

[11] John A Elefteriades. Natural history of thoracic aortic aneurysms: indications for surgery, and surgical versus nonsurgical risks. The Annals of thoracic surgery, 74(5):S1877-S1880, 2002.

[12] T Christian Gasser, Ray W Ogden, and Gerhard A Holzapfel. Hyperelastic modelling of arterial layers with distributed collagen fibre orientations. Journal of the Royal Society, Interface, 3:15-35, 2006. 
[13] Katia Genovese, Luciana Casaletto, Jay D Humphrey, and Jia Lu. Digital image correlation-based pointwise inverse characterization of heterogeneous material properties of gallbladder in vitro. Proc. R. Soc. A, 470:20140152, 2014.

[14] Gerhard A Holzapfel and Ray W Ogden. Constitutive modelling of arteries. Proceedings of the Royal Society A: Mathematical, Physical and Engineering Sciences, 466:1551-1597, 2010.

[15] J. D. Humphrey and G. A. Holzapfel. Mechanics,mechanobiology,andmodelingofhumanabdominalaorta and aneurysms. Journal of Biomechanics, 45:805-814, 2012.

[16] Dimitrios C Iliopoulos, Rejar P Deveja, Eleftherios P Kritharis, Despina Perrea, George D Sionis, Konstantinos Toutouzas, Christodoulos Stefanadis, and Dimitrios P Sokolis. Regional and directional variations in the mechanical properties of ascending thoracic aortic aneurysms. Medical engineering \& physics, 31(1):1-9, 2009.

[17] Gunnar Johansson, Ulf Markström, and Jesper Swedenborg. Ruptured thoracic aortic aneurysms: a study of incidence and mortality rates. Journal of vascular surgery, 21(6):985-988, 1995.

[18] Jeffrey A Jones, Christy Beck, John R Barbour andJouzas A Zavadzkas, Rupak Mukherjee, Francis G Spinale, and John S Ikonomidis. Alterations in aortic cellular constituents during thoracic aortic aneurysm development. The American Journal of Pathology, 175:1746-1756, 2009.

[19] Khalil Khanafer, Ambroise Duprey, Mohammad Zainal, Marty Schlicht, David Williams, and Ramon Berguer. Determination of the elastic modulus of ascending thoracic aortic aneurysm at different ranges of pressure using uniaxial tensile testing. The Journal of thoracic and cardiovascular surgery, 142(3):682686, 2011.

[20] J. Lu and X. Zhao. Pointwise identification of elastic properties in nonlinear hyperelastic membranes. Part I: Theoretical and computational developments. ASME Journal of Applied Mechanics, 76:061013/1061013/10, 2009.

[21] Jia Lu, Xianlian Zhou, and Madhavan L Raghavan. Inverse method of stress analysis for cerebral aneurysms. Biomechanics and modeling in mechanobiology, 7(6):477-86, December 2008.

[22] Caitlin Martin, Wei Sun, Thuy Pham, and John Elefteriades. Predictive biomechanical analysis of ascending aortic aneurysm rupture potential. Acta biomaterialia, 9(12):9392-9400, 2013. 
[23] Takeo Matsumoto, Tomohiro Fukui, Toshihiro Tanaka, Naoko Ikuta, Toshiro Ohashi, Kiichiro Kumagai, Hiroji Akimoto, Koichi Tabayashi, and Masaaki Sato. Biaxial tensile properties of thoracic aortic aneurysm tissues. Journal of Biomechanical Science and Engineering, 4(4):518-529, 2009.

[24] Ruth J Okamoto, Jessica E Wagenseil, William R DeLong, Sara J Peterson, Nicholas T Kouchoukos, and Thoralf M Sundt III. Mechanical properties of dilated human ascending aorta. Annals of biomedical engineering, 30(5):624-635, 2002.

[25] Thuy Pham, Caitlin Martin, John Elefteriades, and Wei Sun. Biomechanical characterization of ascending aortic aneurysm with concomitant bicuspid aortic valve and bovine aortic arch. Acta biomaterialia, 9(8):7927-7936, 2013.

[26] Joseph E Pichamuthu, Julie A Phillippi, Deborah A Cleary, Douglas W Chew, John Hempel, David A Vorp, and Thomas G. Gleason. Differential tensile strength and collagen composition in ascending aortic aneurysms by aortic valve phenotype. 96:2147-2154, 2013.

[27] Madhavan L Raghavan, Jarin Kratzberg, Erasmo Magalhães Castro de Tolosa, Mauro M Hanaoka, Patricia Walker, and Erasmo Simão da Silva. Regional distribution of wall thickness and failure properties of human abdominal aortic aneurysm. Journal of biomechanics, 39(16):3010-3016, 2006.

[28] ML Raghavan, Marshall W Webster, and David A Vorp. Ex vivo biomechanical behavior of abdominal aortic aneurysm: assessment using a new mathematical model. Annals of biomedical engineering, 24(5):573-582, 1996.

[29] Aaron Romo, Pierre Badel, Ambroise Duprey, Jean-Pierre Favre, and Stéphane Avril. In vitro analysis of localized aneurysm rupture. Journal of biomechanics, 47(3):607-616, 2014.

[30] Shukei Sugita and Takeo Matsumoto. Yielding phenomena of aortic wall and intramural collagen fiber alignment: Possible link to rupture mechanism of aortic aneurysms. Journal of Biomechanical Science and Engineering, 8(2):104-113, 2013.

[31] Shukei Sugita, Takeo Matsumoto, Toshiro Ohashi, Kiichiro Kumagai, Hiroji Akimoto, Koichi Tabayashi, and Masaaki Sato. Evaluation of rupture properties of thoracic aortic aneurysms in a pressure-imposed test for rupture risk estimation. Cardiovascular Engineering and Technology, 3(1):41-51, 2012. 
[32] Jonathan P Vande Geest, David HJ Wang, Stephen R Wisniewski, Michel S Makaroun, and David A Vorp. Towards a noninvasive method for determination of patient-specific wall strength distribution in abdominal aortic aneurysms. Annals of biomedical engineering, 34(7):1098-1106, 2006.

[33] D. A. Vorp, M. L. Raghavan, S. C. Muluk, M. S. Makaroun, D. L. Steed, R. Shapiro, and M. W. Webster. Wall strength and stiffness of aneurysmal and nonaneurysmal abdominal aorta. In Abdominal Aortic Aneurysm: Genetics, Pathophysiology, and Molecular Biology, volume 800 of Annals of the New York Academy of Sciences, pages 274-276. 1996.

[34] David A Vorp, Brian J Schiro, Marek P Ehrlich, Tatu S Juvonen, M Arisan Ergin, and Bartley P Griffith. Effect of aneurysm on the tensile strength and biomechanical behavior of the ascending thoracic aorta. The Annals of thoracic surgery, 75(4):1210-1214, 2003.

[35] X. Zhao, X. Chen, and J. Lu. Pointwise identification of elastic properties in nonlinear hyperelastic membranes. Part II: Experimental validation. ASME Journal of Applied Mechanics, 76:061014/1-061014/8, 2009.

[36] X. Zhao, M. L. Raghavan, and J. Lu. Characterizing heterogeneous properties of cerebral aneurysms with unknown stress-free geometry: A precursor to in vivo identification. Journal of Biomechanical Engineering, 133:051008-1/051008/11, 2011. 


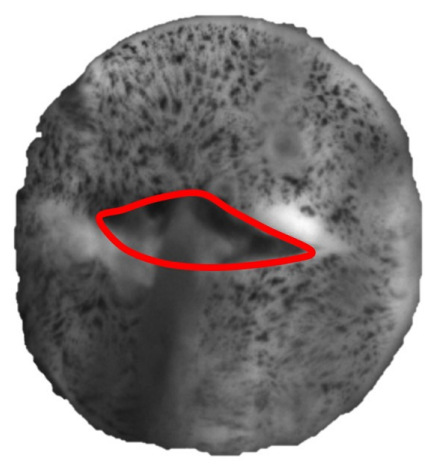

(a) Patient 1: Orifice image

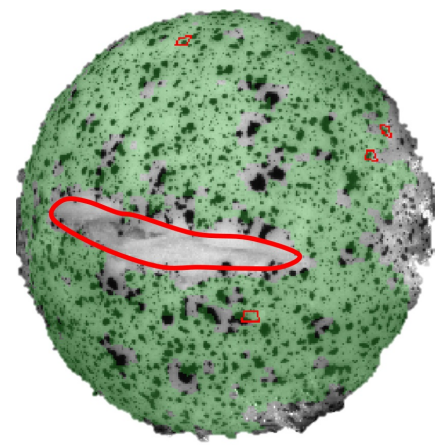

(e) Patient 5(a): Orifice image

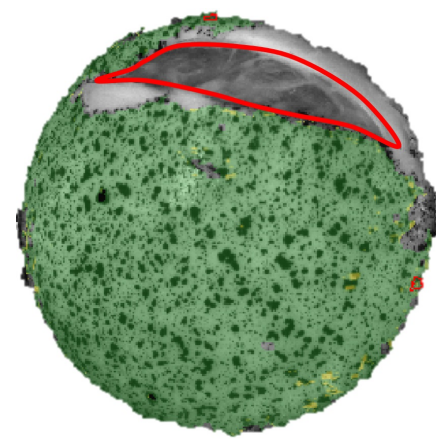

(i) Patient 6: Orifice image

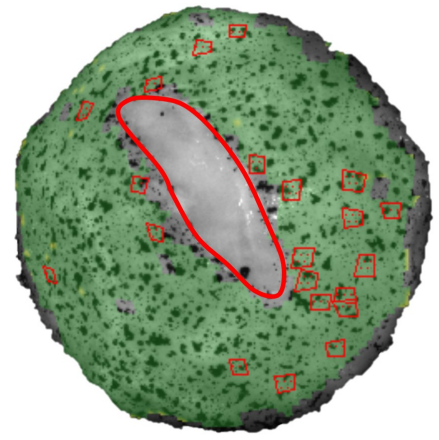

(m) Patient 7(b): Orifice image

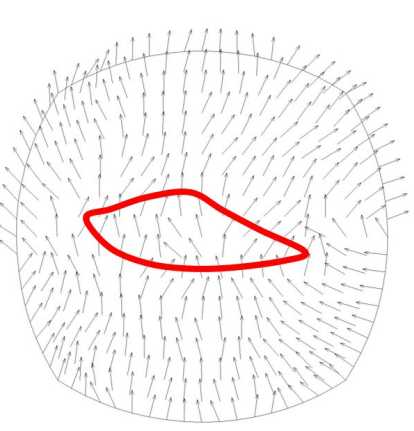

(b) Patient 1: Fiber direction

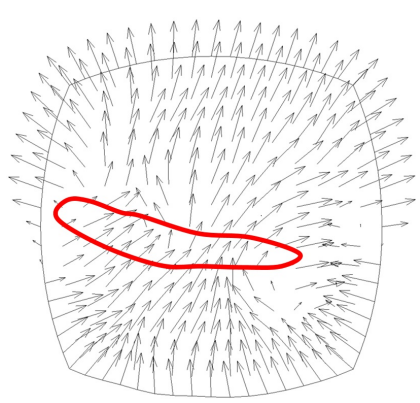

(f) Patient 5(a): Fiber direction

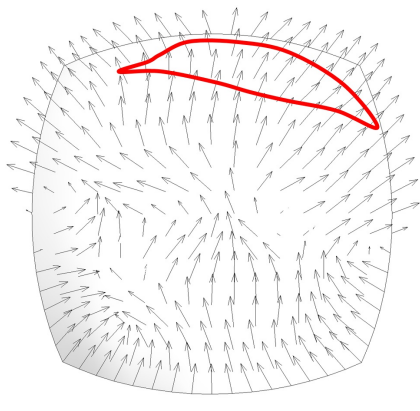

(j) Patient 6: Fiber direction

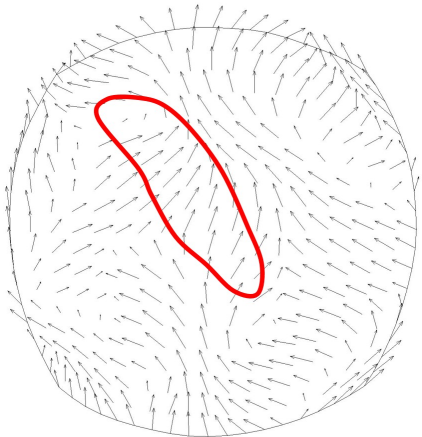

(n) Patient 7(b): Fiber direction

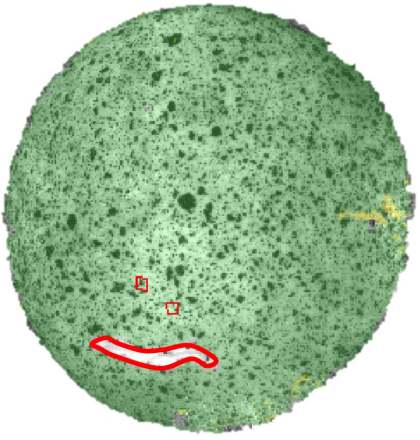

(c) Patient 2: Orifice image

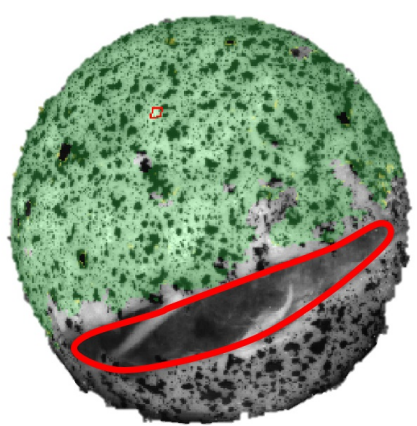

(g) Patient 5(b): Orifice image

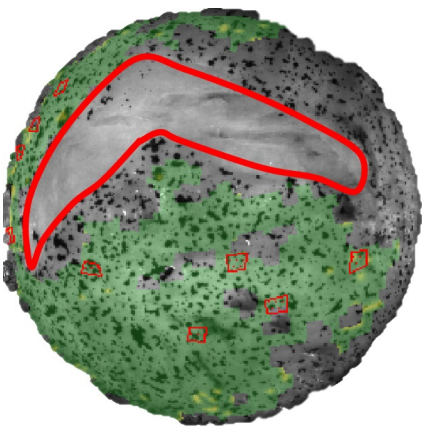

(k) Patient 7(a): Orifice image

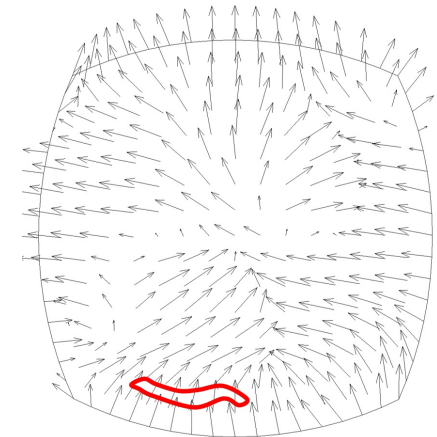

(d) Patient 2: Fiber direction

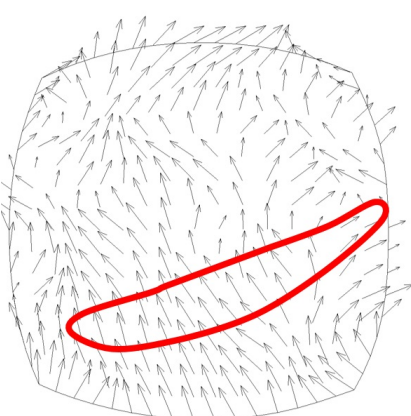

(h) Patient 5(b): Fiber direction

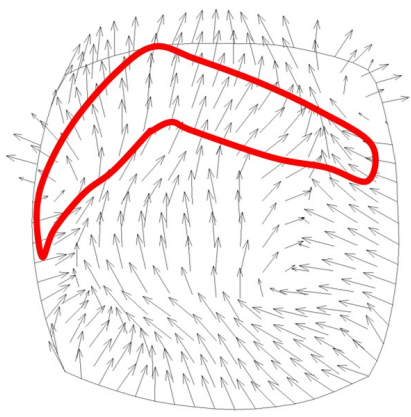

(l) Patient 7(a): Fiber direction

Figure 9: Orifice orientation and predicted fiber direction 


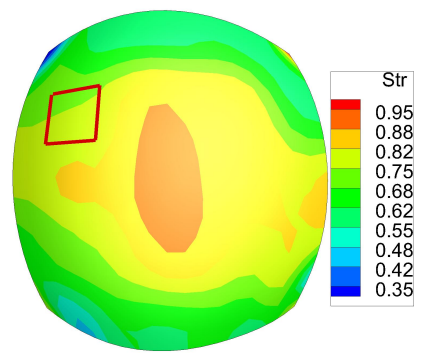

(a) Patient 3: Tension

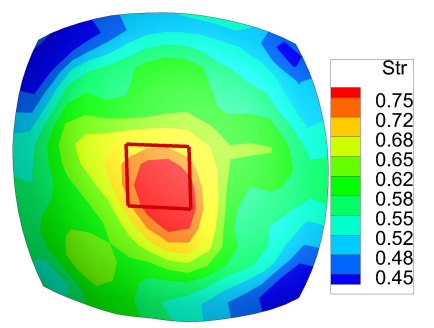

(e) Patient 5(a): Tension

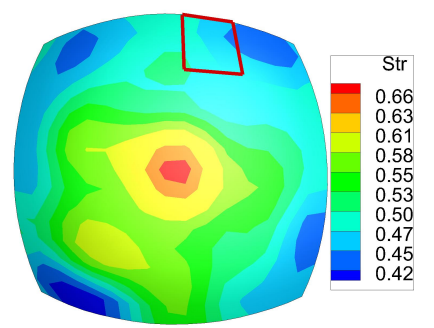

(i) Patient 6: Tension

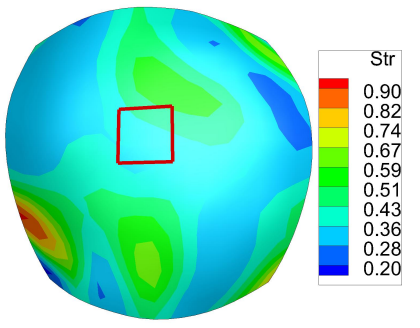

(m) Patient 7(b): Tension

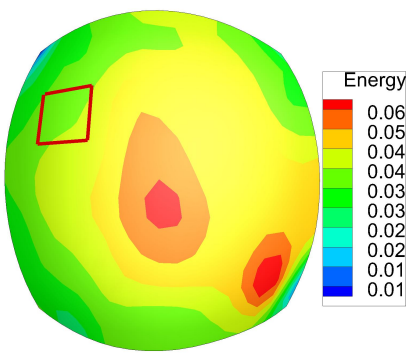

(b) Patient 3: Energy

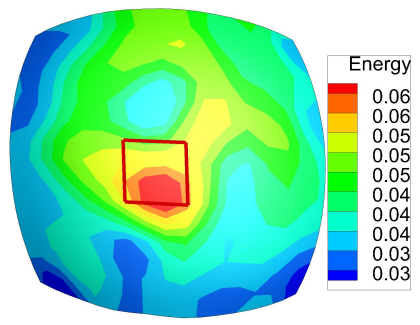

(f) Patient 5(a): Energy

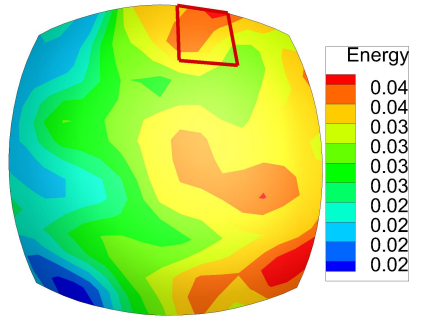

(j) Patient 6: Energy

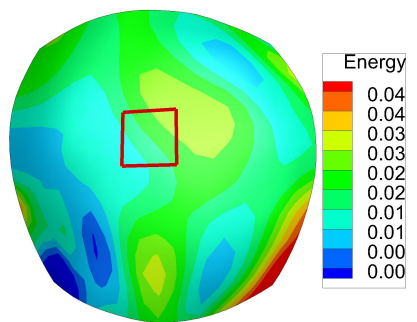

(n) Patient 7(b): Energy

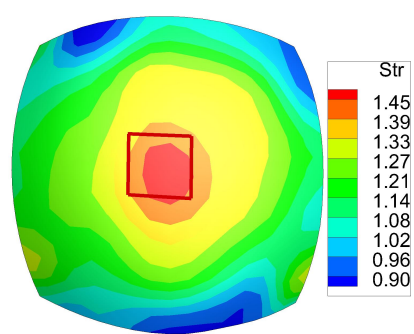

(c) Patient 4: Tension

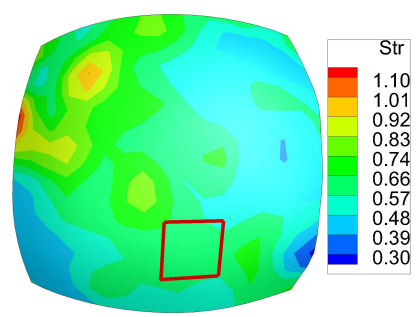

(g) Patient 5(b): Tension

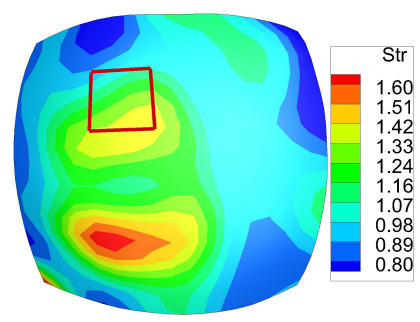

(k) Patient 7a: Tension

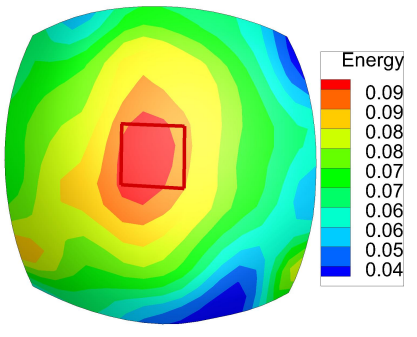

(d) Patient 4: Energy

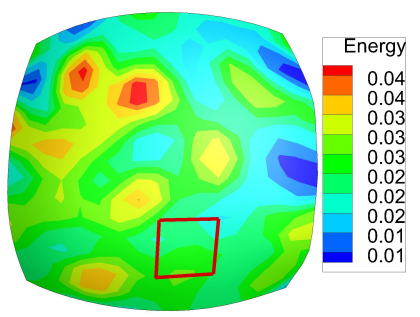

(h) Patient 5(b): Energy

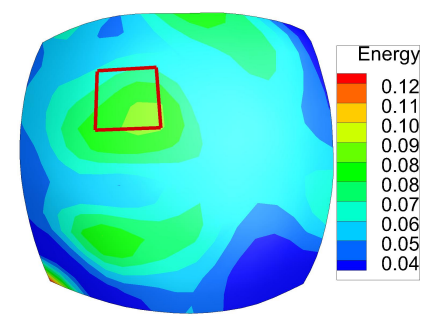

(1) Patient 7(a): Energy

Figure 10: Rupture location and the sites of the peak tension and peak energy 